Technical note

\title{
Short-duration dynamic FDG PET imaging: Optimization and clinical application
}

\author{
Rezvan Samimi ${ }^{\text {a }}$, Alireza Kamali-Asl ${ }^{\text {a, *, Parham Geramifar }}{ }^{\text {b,*, Jörg van den Hoff }}{ }^{\text {c,d }}$, \\ Arman Rahmim ${ }^{\text {eff }}$ \\ ${ }^{a}$ Department of Medical Radiation Engineering, Shahid Beheshti University, Tehran, Iran \\ ${ }^{\mathrm{b}}$ Research Center for Nuclear Medicine, Shariati Hospital, Tehran University of Medical Sciences, Tehran, Iran \\ ${ }^{c}$ PET Center, Institute of Radiopharmaceutical Cancer Research, Helmholtz-Zentrum Dresden-Rossendorf, Dresden 01328, Germany \\ ${ }^{\mathrm{d}}$ Department of Nuclear Medicine, University Hospital Carl Gustav Carus, Technische Universität Dresden, Dresden 01307, Germany \\ ${ }^{\mathrm{e}}$ Departments of Radiology and Physics, University of British Columbia, Vancouver, BC, Canada \\ ${ }^{\mathrm{f}}$ Department of Radiology and Radiological Science, Johns Hopkins University, Baltimore, MD, USA
}

\section{A R T I C L E I N F O}

\section{Keywords:}

\section{Dynamic PET}

Kinetic modelling

GATE

STIR

FDG PET

\begin{abstract}
A B S T R A C T
Purpose: We aimed to investigate whether short dynamic PET imaging started at injection, complemented with routine clinical acquisition at 60-min post-injection (static), can achieve reliable kinetic analysis.

Methods: Dynamic and static 18F-2-fluoro-2-deoxy-D-glucose (FDG) PET data were generated using realistic simulations to assess uncertainties due to statistical noise as well as bias. Following image reconstructions, kinetic parameters obtained from a 2-tissue-compartmental model (2TCM) were estimated, making use of the static image, and the time duration of dynamic PET data were incrementally shortened. We also investigated, in the first 2-min, different frame sampling rates, towards optimized dynamic PET imaging. Kinetic parameters from shortened dynamic datasets were additionally estimated for 9 patients (15 scans) with liver metastases of colorectal cancer, and were compared with those derived from full dynamic imaging using correlation and Passing-Bablok regression analyses.

Results: The results showed that by reduction of dynamic scan times from 60-min to as short as 5-min, while using static data at 60-min post-injection, bias and variability stayed comparable in estimated kinetic parameters. Early frame samplings of 5, 24 and 30 s yielded highest biases compared to other schemes. An early frame sampling of $10 \mathrm{~s}$ generally kept both bias and variability to a minimum. In clinical studies, strong correlation ( $\mathrm{r} \geq 0.97, \mathrm{P}<$ 0.0001 ) existed between all kinetic parameters in full vs. shortened scan protocols.

Conclusions: Shortened 5-min dynamic scan, sampled as $12 \times 10+6 \times 30 \mathrm{~s}$, followed by 3-min static image at 60 min post-injection, enables accurate and robust estimation of 2TCM parameters, while enabling generation of SUV estimates.
\end{abstract}

\section{Introduction}

The standardized uptake value (SUV) is nowadays routinely employed in single temporal frame (static) PET imaging for diagnosis, staging and treatment response monitoring of a wide range of oncologic malignancies [1]. At the same time, PET tracer distribution is a dynamic process and a number of factors can affect SUV values [2].

As long as no detailed tracer kinetic analysis is intended to differentiate between the different pharmacokinetic parameters $\left(\mathrm{K}_{1}, \mathrm{k}_{2}, \mathrm{k}_{3}\right.$, $\mathrm{k}_{4}, \mathrm{~V}_{\mathrm{B}}$ in the case of FDG), the recently proposed tumor to blood standard uptake ratio (SUR) has been shown to be able to overcome most of the shortcomings of SUV and to act as a superior surrogate of the metabolic uptake rate $\mathrm{K}_{\mathrm{i}}$ [3]. However, like SUV, SUR is derived from a static acquisition at late times and thus does not allow to draw any conclusions regarding the relative contribution of unidirectional inflow $\left(\mathrm{K}_{1}\right)$, early tissue clearance $\left(\mathrm{k}_{2}\right)$, phosphorylation $\left(\mathrm{k}_{3}\right)$, dephosphorylation $\left(\mathrm{k}_{4}\right)$ and the tissue blood fraction $\left(\mathrm{V}_{\mathrm{B}}\right)$ to the observed tracer uptake.

Alternatively, dynamic PET imaging, employed mostly in the research setting, is able to track PET radiotracer biodistribution in the body over time, offering the possibility of further analysis including full

\footnotetext{
* Corresponding authors at: Department of Medical Radiation Engineering, Shahid Beheshti University, Tehran, Iran and Research Center for Nuclear Medicine, Shariati Hospital, Tehran University of Medical Sciences, Tehran, Iran.

E-mail addresses: a_kamali@sbu.ac.ir (A. Kamali-Asl), pgeramifar@tums.ac.ir (P. Geramifar).
} 
kinetic modelling and potentially enhanced clinical tasks [4]. Some early studies have shown that dynamic quantitative measurements are superior to static acquisition in differentiating benign from malignant lung lesions specially in patients with borderline SUVs $[5,6]$. Furthermore, kinetic analysis can be particularly helpful in therapy response monitoring [7].

Despite these potential advantages, dynamic imaging is not used routinely in the clinic. This is related to the long scanning time and the difficulty of blood sampling [8]. Moreover, patients cannot remain immobile for a long duration such as 60-min, which has been typically used in past dynamic PET scans. Finally, this significantly degrades the daily throughput of PET scanners.

Several attempts have been made to reduce duration of dynamic PET scans. In a comparison study by Torizuka et al. [9] on 20 patients with primary lung cancers, it was shown that a shorter 30-min dynamic PET acquisition may yield essentially the same results for compartmental modeling as the standard 60-min imaging protocol. Visser et al. [10] used Patlak analysis on 13 patients with non-small-cell lung carcinoma (NSCLC) and noted that a shortened data acquisition protocol of 30-min showed good agreement with a protocol of 50-min. In a brain glioma investigation, Monden et al. [11] demonstrated that a 40-min FDG PET examination provided acceptable estimation of parametric $\mathrm{k}_{3}$ and $\mathrm{K}_{\mathrm{i}}$ images. These studies evaluated the kinetic parameters when duration of PET data acquisition was shortened. Alternatively, some studies involving optimization of frame sampling were conducted in full dynamic PET imaging [12,13]. Moreover, Häggström et al. [14] investigated sampling of the first two minutes in a full dynamic brain study, with frame duration ranging from 1 to $15 \mathrm{~s}$, and found that an early frame duration of 6-15 s minimized overall bias in pharmacokinetic parameters. In any case, these latter studies evaluated full dynamic acquisitions of 60 or 120 min durations, and the influence of chosen frame duration in shortened dynamic PET were not investigated.

The aim of the present study was to investigate whether early dynamic PET, supplemented by 3-minute-per-bed routine clinical wholebody PET data acquired at 60-min post-injection, can achieve reliable kinetic analysis. To obtain a potentially shortened dynamic PET protocol, the following two questions needs to be investigated: first, the necessary duration of the initial dynamic PET acquisition required for accurate kinetic analysis; secondly, how the frame duration (especially of early frames around the blood peak) affects estimation of the kinetic parameters. As such, in the present study, the effects of decreasing dynamic PET duration as well as the chosen framing scheme on compartmental modelling analysis were investigated, and a novel shortened dynamic PET acquisition protocol was proposed enabling robust kinetic analysis with the aid of clinical static PET data at 60 -min post-injection.

\section{Materials and methods}

As elaborated later in this section, we first used realistic non-uniform rational B-spline (NURB) cardiac and torso (NCAT) anthropomorphic phantom images in combination with simulations using the open source Software for Tomographic Image Reconstruction (STIR) to generate realistic PET sinogram data and reconstructed images. Our analytic simulations were validated using Monte Carlo (MC) simulations. Kinetic parameters were then estimated with the inclusion of a late static image, while the time duration of early dynamic PET data were shortened and compared with those derived from full dynamic PET images. We also investigated the impact of different early frame durations (dynamic sampling rates) on bias and variability in kinetic parameters as obtained from shortened dynamic PET data towards optimal imaging protocols. Subsequently, shortened dynamic protocol was evaluated in a group of 9 patients with liver metastases of colorectal cancer ( 1 tumor/patient) for which 15 fully dynamic investigations were available.

\subsection{Simulation study}

The NCAT phantom [15] was used to generate transmission and emission images, incorporating time-dependent activity maps for organ tissues and a spherical lung tumor (24 $\mathrm{mm}$ in diameter). The phantom had voxel sizes of $2.25 \times 2.25 \times 2.425 \mathrm{~mm}$ and is depicted in the left side of Fig. 1.

A 2-tissue compartmental model (2TCM) with irreversible tracer metabolism $\left(\mathrm{k}_{4} \simeq 0\right)$ is commonly employed to describe FDG kinetics. [4]. Tissue time activity curves (tTACs) were generated based on 2TCM, plasma input function (IF) based on the literature [8] and FDG kinetic physiological parameters (mean value of 9 patients with NSCLC) presented in Table 1 [16].

The simulated TACs for all tissues in the phantom are shown in Fig. 1. Then, the generated TACs were assigned to each phantom region to produce a time series of activity maps which were later passed to analytical and MC simulations using the simulated ECAT EXACT HR+ (CTI/Siemens Knoxville) [17].

\subsubsection{Analytic simulations}

FDG distribution images were generated for single-bed dynamic PET scan of the thorax spanning 60-min followed by standard static PET (60 to $63 \mathrm{~min}$ ). This was followed by generation of projection data using the STIR software based on ray tracing [18]. Attenuation coefficient projection data were estimated from the attenuation images. The effect of scatter was simulated analytically using the single scatter simulation (SSS) formula. Finally, three different Poisson noise levels (low, medium and high) were applied. These dynamic PET data were reconstructed using STIR into dynamic image sets with frames at $60 \times 10 \mathrm{~s}, 10 \times 60 \mathrm{~s}$, $20 \times 120$ s making a total of 60 -min followed by 3 -min static image 60 min after injection.

\subsubsection{Monte Carlo simulations}

To validate the accuracy and signal-to-noise values obtained from the analytic method, we needed a more realistic study with minimal approximations. To do this, we used Geant4 Application for Tomography Emission (GATE) [19], an open source and a well validated MC simulation package. Our simulation was comprised of an initial 10-min single-bed dynamic scan of thorax followed by the 3-min conventional static scan at 60 -min post-injection. The activity of each phantom organ was read from the generated TACs and was updated every second of the simulation for the first 10-min, where changes in the activity were very high. All sinogram data were subsequently reconstructed into 60 frames each 10-sec in duration, and a final 3-min static image. To obtain the calibration factor, a homogeneous, $80 \mathrm{~mm}$ radius cylindrical source in water was simulated with $63 \mathrm{MBq}{ }^{18}$ F-FDG for about 15 -min. The main drawback of MC method is the long execution times especially for full dynamic scans. Our MC simulations required about $6800 \mathrm{CPU}$ hours but the actual time was faster as the simulation was performed using high performance cluster computing at our university.

\subsubsection{Image reconstruction}

Image reconstructions of raw data from both analytic and GATE simulations were performed using the 3D ordered subsets maximum likelihood expectation maximization (OSEM) algorithm as implemented in the STIR package, involving 4 iterations and 4 subsets. The attenuation maps relevant for $511 \mathrm{keV}$ photons were used for attenuation correction. For GATE simulation, the known distributions of simulated scatter and random events were used for correction, whereas the scatter correction was performed based on SSS algorithm for analytic simulation. It was reported by Häggström et al. that both scatter correction schemes worked equally well [20].

\subsection{Clinical study}

We additionally evaluated 9 patients with liver metastases of 

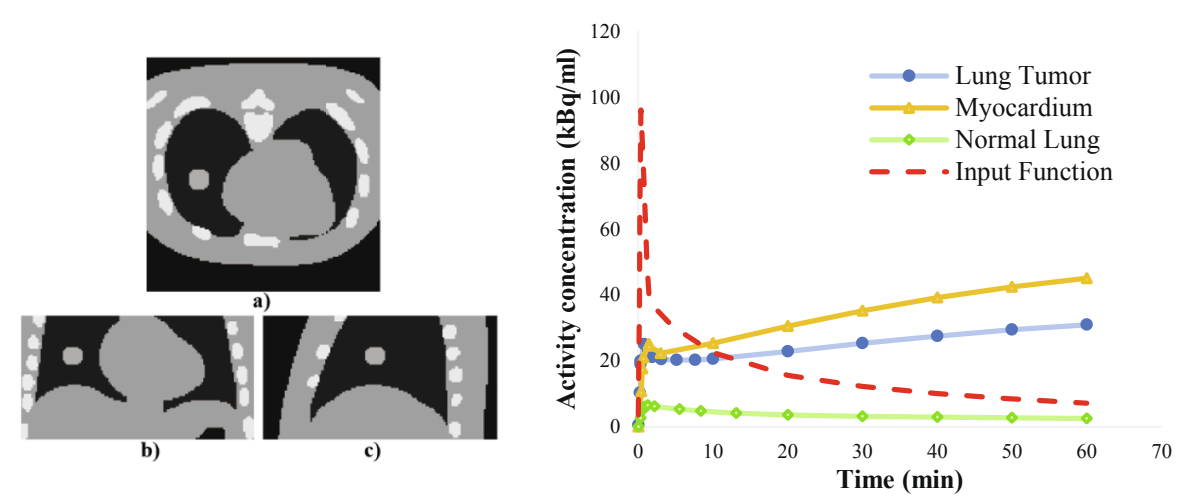

Fig. 1. (left) The voxelized NCAT phantom with a spherical lung tumor (24 mm in diameter) used in the simulations. a) transverse, b) coronal and c) sagittal views of the attenuation map. (right) The IF and generated noise-free tTACs.

Table 1

Simulated kinetic parameter values for FDG $[8,16]$.

\begin{tabular}{llllll}
\hline Regions & $\mathrm{K}_{1}(1 / \mathrm{min})$ & $\mathrm{k}_{2}(1 / \mathrm{min})$ & $\mathrm{k}_{3}(1 / \mathrm{min})$ & $\mathrm{V}_{\mathrm{B}}$ & $\mathrm{K}_{\mathrm{i}}(1 / \mathrm{min})$ \\
\hline Myocardium & 0.6 & 1.2 & 0.1 & 0 & 0.04615 \\
Normal Lung & 0.108 & 0.735 & 0.0016 & 0.0017 & 0.0023 \\
Lung Tumor & 0.301 & 0.864 & 0.097 & 0.168 & 0.03038 \\
\hline
\end{tabular}

colorectal cancer (mean age: 62.8 years, range [48-76]; mean weight $85.5 \mathrm{~kg}$, range [73-100]). For each patient, 1-3 dynamic PET scans of 60-min in duration were performed (altogether 15 scans). Retrospective PET data available were collected from the ECAT EXACT HR + scanner (Siemens/CTI, Knoxville, TN, USA) immediately following injection of 346 to 430 MBq FDG. This scanner consist of BGO scintillators (32 rings, 576 crystals/ring), with an axial field of view of $15.5 \mathrm{~cm}$, while data acquisition was performed in $3 \mathrm{D}$ mode. The acquired data were sorted into 23 to 31 frames with 10 to $20 \mathrm{~s}$ duration during bolus passage, 30 to $150 \mathrm{~s}$ duration until 10-min post-injection, and $300 \mathrm{~s}$ duration afterwards. PET images were reconstructed using attenuation-weighted OSEM reconstruction (6 iterations, 16 subsets, $6 \mathrm{~mm}$ FWHM Gaussian filter).

\subsection{Data analysis}

Quantitative evaluation using simulation studies was based on volume of interests (VOIs), which were delineated on each PET image using the AMIDE open source software [21]. The IF was image-derived utilizing spherical VOIs with $15 \mathrm{~mm}$ diameter in the left ventricle and 10 $\mathrm{mm}$ diameter in the atrium while maintaining an adequate distance from myocardium, then averaging them; and the peak height was consistent with the highest of all peaks according to Hoekstra et al. [22]. VOIs are shown in Supplementary Fig. S1. Tumor VOIs were delineated in the last time frame of the PET image using thresholding by $50 \%$ of the maximum intensity and were then copied to all frames. Kinetic parameters of the lung tumors were then estimated using 2TCM through an inhouse code. To generate shortened acquisitions, every time we incrementally omitted data of the last dynamic frame. Then, to predict tTACs as well as IF in omitted time points we utilized the routine static image data acquired at 60-min post-injection; for this purpose, a bi-exponential function was fitted to the dynamic plus the static data, as sums of exponential functions can describe the tail of the plasma TAC using a population-based IF [23]. We wish to point out that other reasonable models of IF (e.g., inverse power law) might also depict reasonable performance.

The choice of the optimal scan duration was based on quantitative criteria of bias and variability (standard deviation (SD) of estimated kinetic parameters from multiple noise realizations). In the simulation study, the results of the shortened acquisitions were compared with the referenced result (60-min dynamic data), and the \%bias, SD and Root Mean Square Error (RMSE) of the kinetic parameters value were calculated.

For patient studies, VOI definition was performed using the Imager4D software [24]. The IF was determined from a spherical VOI of $15 \mathrm{~mm}$ diameter centered in the aorta (Supplementary Fig. S2). Liver tumor TACs were calculated using the mean value of the VOIs in each frame. Kinetic analysis of clinical dynamic PET images was performed using PMOD (version 3.9; PMOD Technologies GmbH). Correlations between the kinetic parameters calculated from each of the two methods compared with the gold standard were assessed by (i) Pearson's (r) to determine relationship, and (ii) Passing-Bablok regression to test for the presence of differences between the two methods [25]. A cumulative sum test for linearity was used to validate the applicability of Passing-Bablok analysis. Random differences between two methods were measured using residual SD. All statistical analysis were performed in MedCalc (version 19.1.3; MedCalc Software bvba).

\subsection{Frame sampling}

The first few minutes within dynamic PET data acquisition have the most rapid variations in tracer uptake and clearance. The blood peak is especially critical in compartmental model fitting which occurs at very early times. To better capture these fast variations and for a better definition of the early blood peak, short frames may be required during the first minutes of a dynamic PET study [14].

Since utilizing higher temporal sampling results in larger noise levels in the individual images, and the trade-off between noise and temporal resolution is higher in the early frames, we aimed to optimize dynamic scan duration based on simulated studies elaborated in section 2.1.1. Subsequently, keeping the total scan duration constant, we investigated the effect of different frame durations for the first 2-min (covering the early blood peak [14]) for optimal estimation of the pharmacokinetic parameters. For this investigation, simulations with multiple noise realizations were performed, including 6 different dynamic framing sequences for the first 2-min of imaging involving: (i) $24 \times 5 \mathrm{~s}$, (ii) $12 \times 10$ $\mathrm{s}$, (iii) $8 \times 15 \mathrm{~s}$, (iv) $6 \times 20 \mathrm{~s}$, (v) $5 \times 24 \mathrm{~s}$, or (vi) $4 \times 30 \mathrm{~s}$. The frame sampling after 2-min was based on the concept of selecting time points where $>5 \%$ decrease in IF activity is observed. The bias and SD were calculated, similar to previous subsection.

\section{Results}

\subsection{Comparison of analytic and GATE simulated dynamic data}

Fig. 2 (top) demonstrates a visual comparison between reconstructions of one of the dynamic frames as well as static dataset after 60-min uptake, from both analytic and GATE simulated data. Fig. 2 
a)

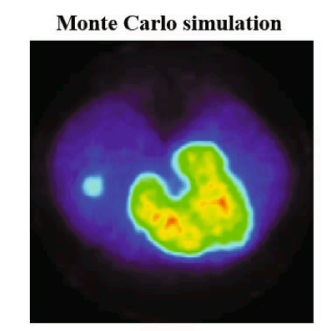

b)
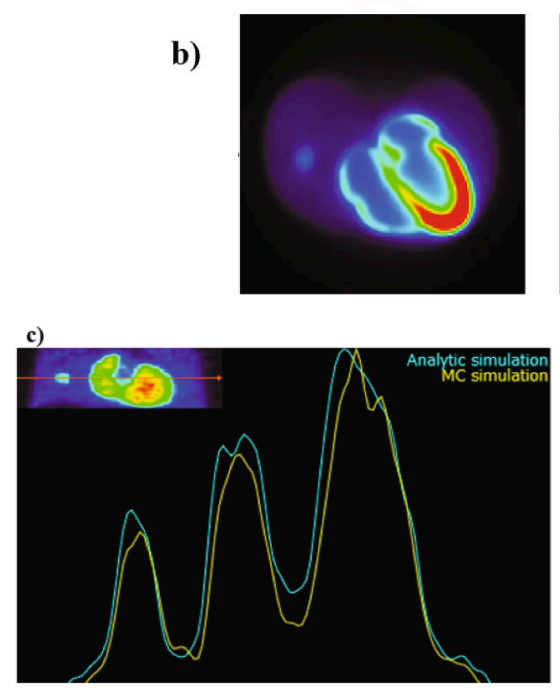

e)

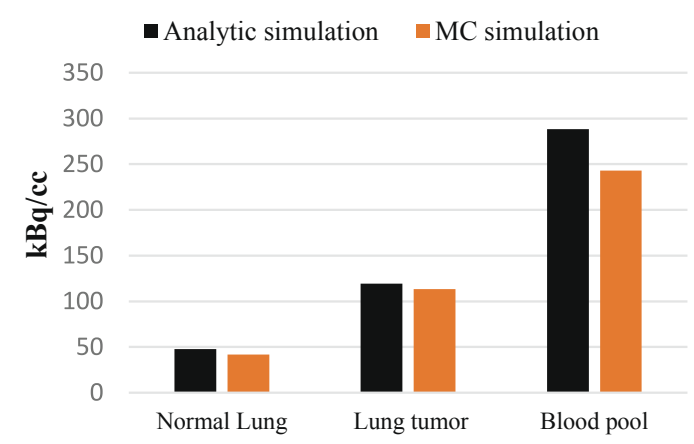

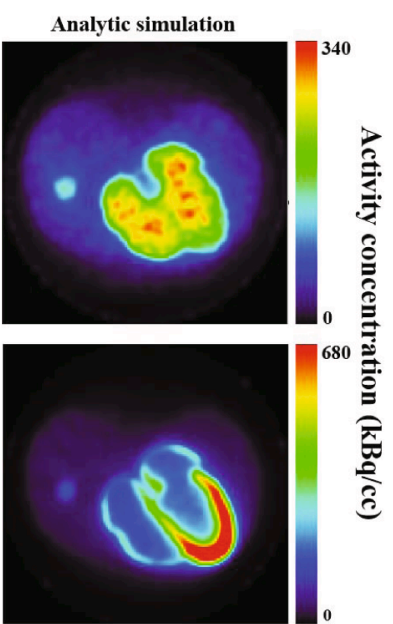

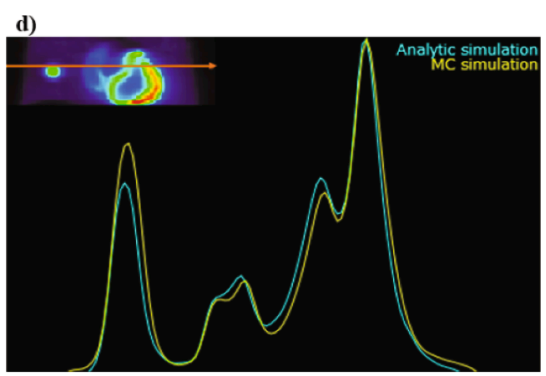

f)

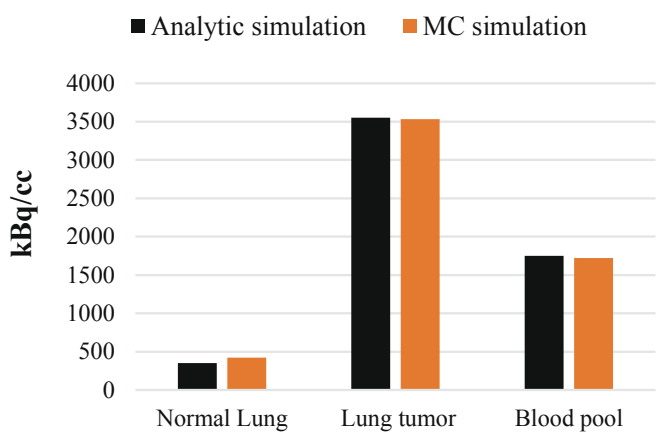

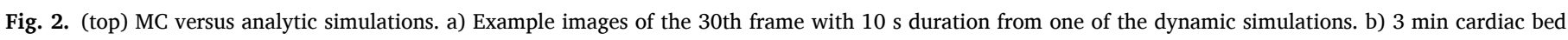

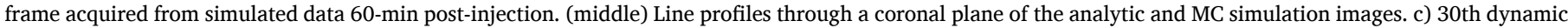

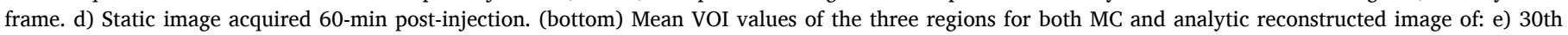
dynamic frame and f) static image acquired 60-min post-injection. * Co-registration was not required as images came from the same digital phantom.

(middle) then illustrates line profiles through a coronal plane of the mentioned reconstructed images, while Fig. 2 (bottom) shows the differences between analytic and GATE simulations for VOI mean values. We show, via visual as well as quantitative comparisons (Fig. 2), that dynamic images with short (10-sec) and long (3-min) frame durations, as obtained from analytic simulations were very similar to those obtained from MC simulations. As such, to speed up our overall investigation, we pursued the use of analytical simulations, instead of GATE MC method.

\subsection{Quantitative assessment in the analytically simulated phantom}

Fig. 3 shows the calculated bias, SD and RMSE of the estimated parameters for lung tumor as a function of the dynamic scan time duration. All estimations were performed incorporating dynamic data as well as post-injection static data as explained in section 2.3. Overall, SD, bias and RMSE of the estimated parameters increased with reduced dynamic data. At the same time, by reduction of the dynamic scan time from 60 to $4 \mathrm{~min}$, the bias between 60 and $5 \mathrm{~min}$ became 3.5\%, 4.5\%, 3.5\%, 5\%, and $4 \%$ for $\mathrm{K}_{1}, \mathrm{k}_{2}, \mathrm{k}_{3}, \mathrm{~V}_{\mathrm{B}}$ and $\mathrm{K}_{\mathrm{i}}$, respectively. The shortest dynamic duration of 5-min yielded an SD of $18.1 \%, 14.1 \%, 9.1 \%, 4.3 \%$ and $14.3 \%$ for $\mathrm{K}_{1}, \mathrm{k}_{2}, \mathrm{k}_{3}, \mathrm{~V}_{\mathrm{B}}$ and $\mathrm{K}_{\mathrm{i}}$, respectively. RMSE for the shortened dynamic protocol was $18.5 \%, 14.8 \%, 9.7 \%, 6.5 \%$, and $14.9 \%$ for $\mathrm{K}_{1}, \mathrm{k}_{2}$, $\mathrm{k}_{3}, \mathrm{~V}_{\mathrm{B}}$ and $\mathrm{K}_{\mathrm{i}}$, respectively.

Subsequently, the effect of different early dynamic frame sampling schemes was investigated for shortened 5-min dynamic data in the first two minutes of the dynamic scan, being complemented with an additional 60-min acquisition data. The results are shown in Supplementary Fig. S3. On average, the 5, 24 and $30 \mathrm{sec}$ schemes yielded significantly larger biases compared to other schemes. In general, the minimum bias, SD and RMSE was found in 10-sec scheme which were $<10 \%$; however, the bias of the sampling schemes of 10 and $15 \mathrm{sec}$ did not differ 
a)
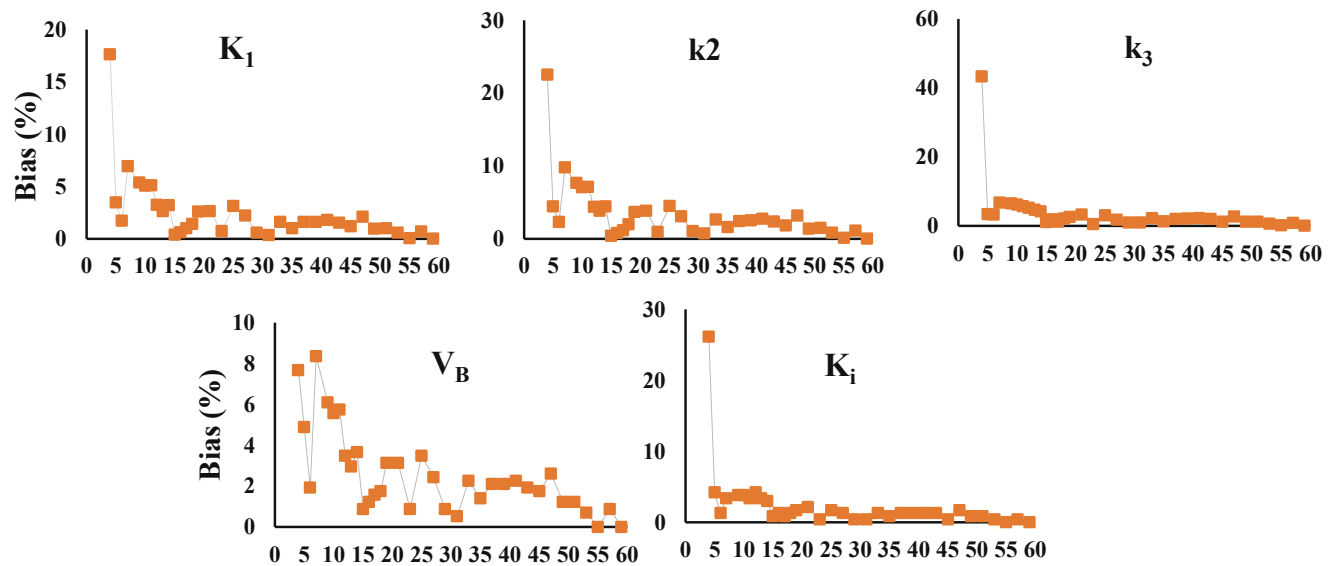

Scan time duration (min)

b)
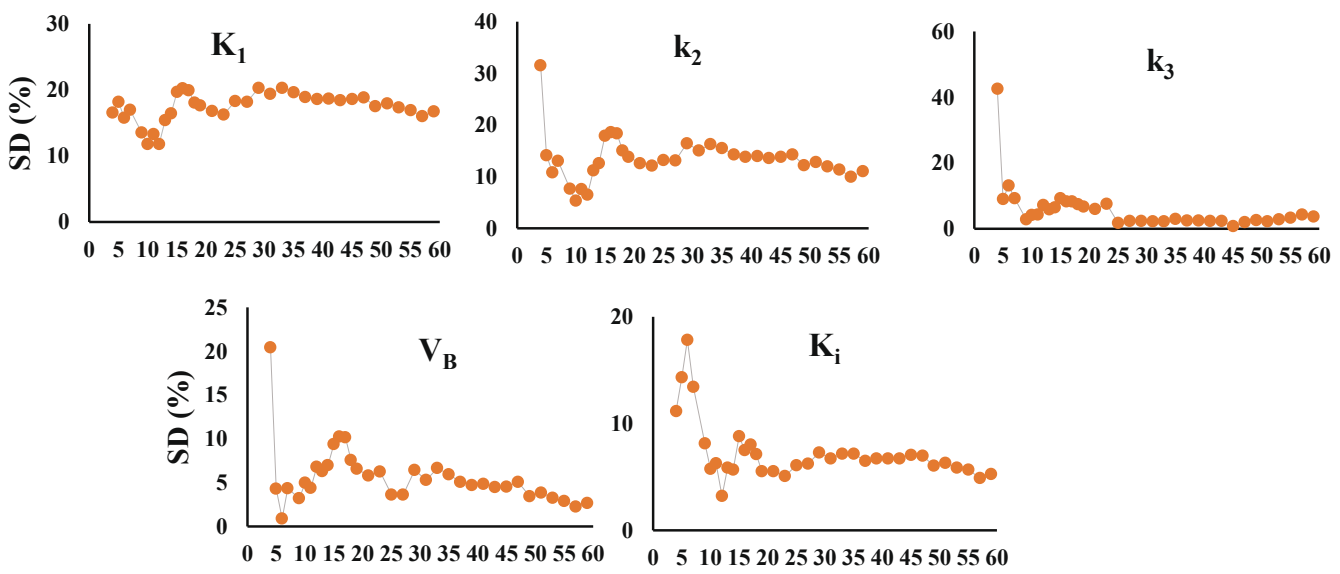

Scan time duration (min)

c)

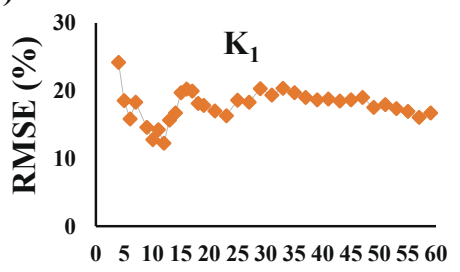

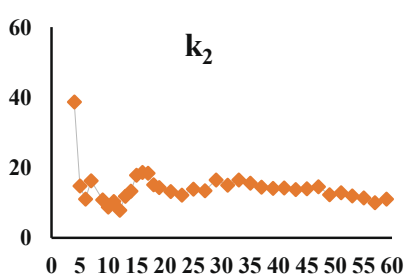

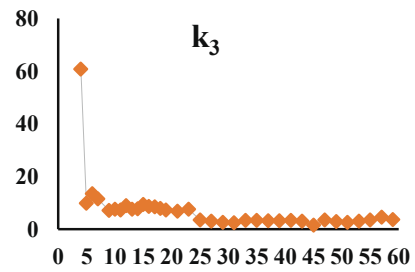

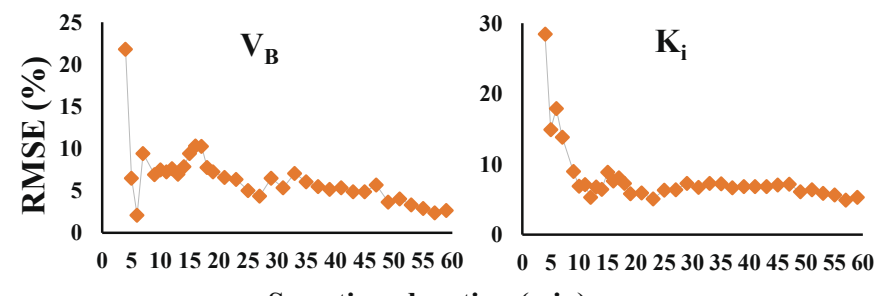

Scan time duration (min)

Fig. 3. a) \%Bias, b) \%SD, and c) RMSE for the 5 estimated micro- and macro-parameters as a function of dynamic scan duration for lung tumor. The smallest duration shown is $4 \mathrm{~min}$; the second time point is $5 \mathrm{~min}$.

significantly. On average, parameter $\mathrm{K}_{\mathrm{i}}$ was the least biased estimate about $7 \%$ for all schemes, and $5 \%$ when excluding the 30 -sec scheme.

The schematic of our proposed protocol is shown in Fig. 4. Shortened dynamic PET/CT imaging was performed via list-mode scanning with bolus injection of FDG, and continued for 5-min for one bed position over cardiac bed. Subsequently, the data were reconstructed as 12 frames of 10 -sec intervals for the first $2 \mathrm{~min}$, followed by $6 \times 30 \mathrm{~s}$. 
Injection

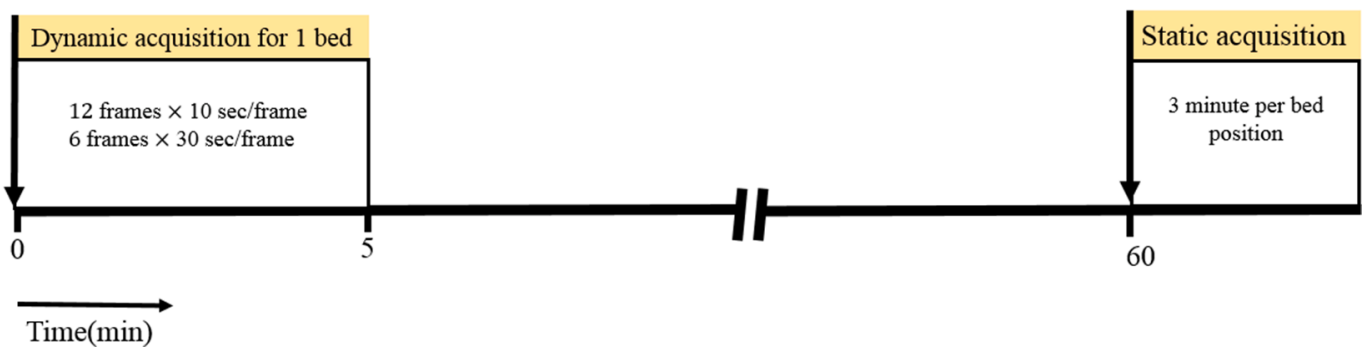

Fig. 4. Schematic representation of the proposed protocol.

\subsection{Clinical evaluation}

For the 9 patients studied (15 scans; 1 tumor/scan), the average bias of the 15 liver lesions between kinetic parameters estimated by full and shortened data as a function of dynamic scan duration were $<10 \%$ for all parameters when the scan time was shortened from 60 to $5 \mathrm{~min}$. The results of correlation and Passing-Bablok analyses between kinetic parameters as calculated using full and shortened data (5-min dynamic + static PET data) are summarized in Table 2. Strong correlations ( $\mathrm{r} \geq$ $0.969, \mathrm{P}<0.0001$ ) were observed between all corresponding parameters. Passing-Bablok regression analyses presented in Fig. 5 showed no significant biases.

\section{Discussion}

The long durations of standard dynamic PET scans limit the use of more sophisticated quantification analysis methods. Despite some efforts on dynamic PET imaging [26,27], the long acquisitions hamper routine usage in the clinic.

To overcome this limitation, we performed kinetic analysis by combining dynamic scanning (immediately following tracer injection) at a single bed focusing on the area of primary interesting pathology, and $\sim 3$-min/bed static imaging 60 -min post-injection as performed routinely. Subsequently, we investigated quantitative performance of kinetic parameters as dynamic PET scan times were shortened. For kinetic parameter estimation, a non-invasive image-derived IF was used. Depending on the location of the pathology, the blood input function can be measured from heart cavities, ascending or descending aorta.

Table 2

Summary of correlations and regressions in the clinical study, between full and shortened data as described in text.

\begin{tabular}{|c|c|c|c|c|c|c|}
\hline \multirow[t]{2}{*}{$\begin{array}{l}\text { Kinetic } \\
\text { parameters }\end{array}$} & \multirow{2}{*}{$\begin{array}{l}\text { Pearson } \\
\text { r (95\% } \\
\text { CI) }\end{array}$} & \multirow[b]{2}{*}{$\mathrm{P}^{*}$} & & \multirow{2}{*}{$\begin{array}{l}\text { Passing- } \\
\text { Bablok } \\
\text { Intercept } \\
(95 \% \mathrm{CI})\end{array}$} & \multirow[b]{2}{*}{$\begin{array}{l}\text { Residual } \\
\text { SD } \\
(95 \% \mathrm{CI})\end{array}$} & \multirow[b]{2}{*}{$\begin{array}{l}\text { Residual } \\
\text { SD\% }\end{array}$} \\
\hline & & & $\begin{array}{l}\text { slope } \\
(95 \% \\
\text { CI) }\end{array}$ & & & \\
\hline $\mathrm{K}_{1}$ & $\begin{array}{l}0.998 \\
(0.995 \\
\text { to } \\
0.999)\end{array}$ & 0.92 & $\begin{array}{l}0.995 \\
(0.971 \\
\text { to } \\
1.033)\end{array}$ & $\begin{array}{l}0.007 \\
(-0.021 \\
\text { to } 0.032)\end{array}$ & $\begin{array}{l}0.0168 \\
(-0.033 \\
\text { to } 0.033)\end{array}$ & $1.8 \%$ \\
\hline $\mathrm{k}_{2}$ & $\begin{array}{l}0.985 \\
(0.956 \\
\text { to } \\
0.995)\end{array}$ & 0.29 & $\begin{array}{l}0.988 \\
(0.917 \\
\text { to } \\
1.063)\end{array}$ & $\begin{array}{l}0.0205 \\
(-0.045 \\
\text { to } 0.098)\end{array}$ & $\begin{array}{l}0.035 \\
(-0.069 \\
\text { to } 0.069)\end{array}$ & $3.6 \%$ \\
\hline $\mathrm{k}_{3}$ & $\begin{array}{l}0.969 \\
(0.908 \\
\text { to } 0.99)\end{array}$ & 0.92 & $\begin{array}{l}1.046 \\
(0.903 \\
\text { to } \\
1.275)\end{array}$ & $\begin{array}{l}-0.002 \\
(-0.009 \\
\text { to } 0.002)\end{array}$ & $\begin{array}{l}0.002 \\
(-0.005 \\
\text { to } 0.005)\end{array}$ & $5.8 \%$ \\
\hline $\mathrm{K}_{\mathrm{i}}$ & $\begin{array}{l}0.969 \\
(0.908 \\
\text { to } 0.99)\end{array}$ & 1 & $\begin{array}{l}1.028 \\
(0.85 \\
\text { to } \\
1.202)\end{array}$ & $\begin{array}{l}-0.001 \\
(-0.006 \\
\text { to } 0.004)\end{array}$ & $\begin{array}{l}0.002 \\
(-0.004 \\
\text { to } 0.004)\end{array}$ & $6.6 \%$ \\
\hline
\end{tabular}

*Cumulative sum test.
Simulations were first performed to assess uncertainties in kinetic parameters due to statistical noise and shortened acquisition times. SD increased with decreased dynamic scan duration. However, $\mathrm{K}_{1}$ was more stable as it mainly governs the early parts of TAC.

Generally decreasing total duration of dynamic scan to 5-min exhibited a bias $<10 \%$ for all kinetic parameters in both simulation and patient studies. In comparison with the parameters obtained from 0 to $60 \mathrm{~min}$ data, we concluded that having at least 5-min dynamic data and a standard clinical static image 60-min post-injection, enable limited biases for all parameters.

This conclusion might be understood as a consequence of the fact that in the early phase the FDG kinetics is essentially describable as a reversible one compartment system since the irreversible pool does not yet contribute sizeable in this phase as long as $\mathrm{k}_{2} \gg \mathrm{k}_{3}$. This condition is in fact fulfilled in all relevant target tissue (see, e.g., Table 1 ). It is then intuitively clear that a scan time with a duration extending a small multiple of $1 / \mathrm{k} 2$ beyond the bolus passage is required to accurately determine this rate constant. Our result that at least 5 min dynamic scans are required is perfectly consistent with this expectation. Analogously, in the area of dynamic whole-body (DWB) imaging [28], there is ongoing investigations as to how short the protocols can be made (even at the cost of quantitative performance) to render such protocols more practical for the clinical setting, while adding value relative to conventional imaging. Overall, such efforts can be first implemented and assessed in specific contexts, such as for monitoring of response to therapy, to arrive at favourable applications that can garner momentum in a clinical setting. We also point out that DWB PET imaging often only invokes Patlak kinetic analysis. In our study, full kinetic analysis can be performed for a single bed while whole-body kinetic analysis can be performed by applying the Patlak model in the rest of the bed positions. Combining Patlak and full compartmental analysis in a predefined pathology-focused single-bed FOV was reported recently in a scanner with larger axial FOV and continuous bed motion [29-31]. We add that our clinical study was performed for the ECAT EXACT HR + scanner, and different performances for shortened protocols might be obtained on newer, higher-sensitivity scanners.

Instead of combining our approach with DWB, it might also be combined with conventional static whole-body imaging and the SUR approach which has been shown to provide a reliable surrogate of Patlak-based Ki determination [3].

Early dynamic PET data contain significant potential towards improved PET-based tasks, such as effective detection of hyperperfusion in hepatocellular carcinoma liver lesions via the first 4-min PET dynamic data [32] and enabling differentiation between clear cell carcinoma and non-clear cell carcinoma of renal tumor with the use of first 10-min data [33]. Furthermore, Mullani et al. [34] showed that the early uptake of ${ }^{18}$ F-FDG (2-min) could be used to measure regional blood flow in tumors in the absence of ${ }^{15} \mathrm{O}$-water.

In addition, we investigated the effect of early frame duration on bias and SD in the proposed protocol. As Häggström et al. [14] have demonstrated the shortest sampling of $1 \mathrm{~s}$ yielded large SD and bias for full dynamic PET scan, thus we evaluated the frame sampling with 

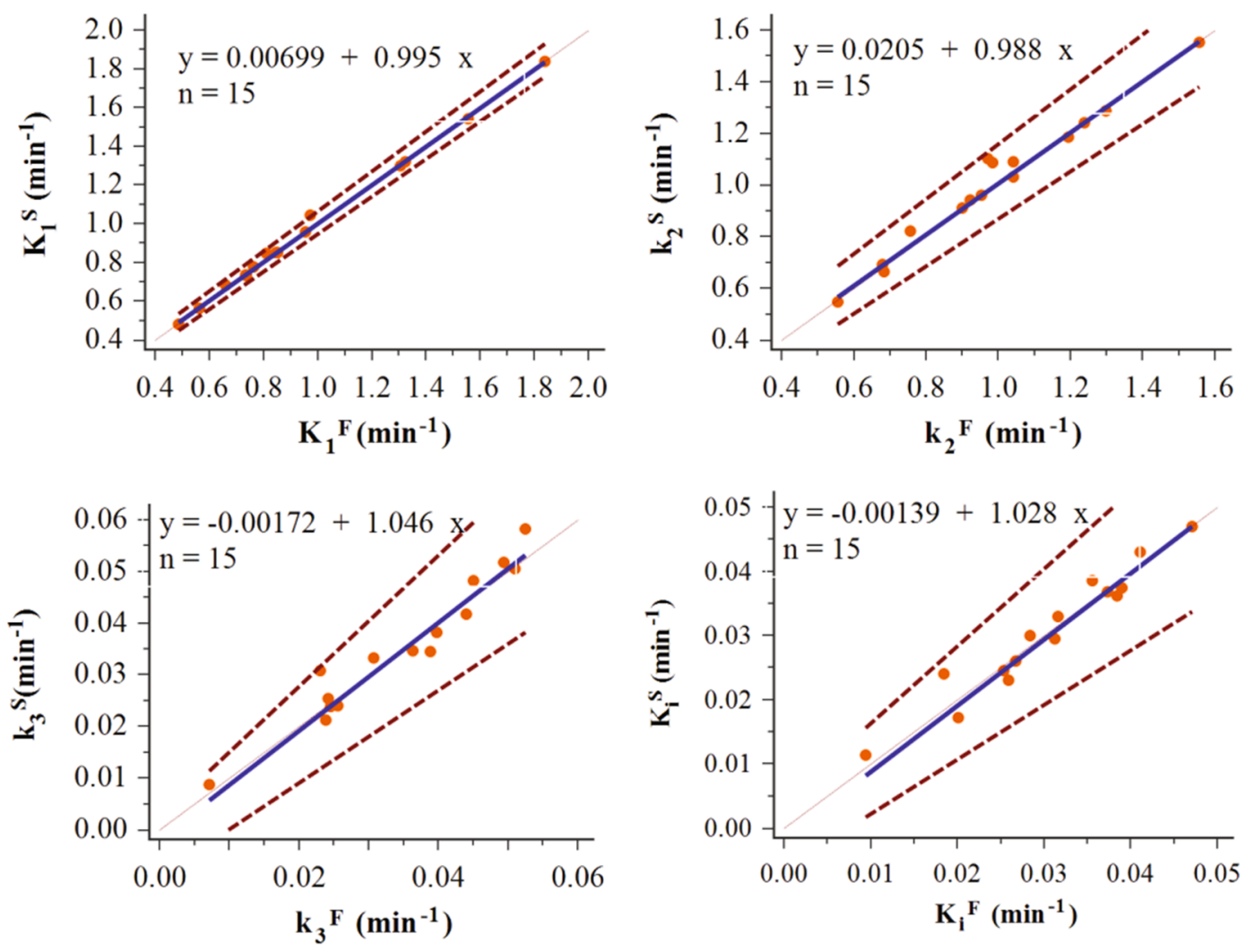

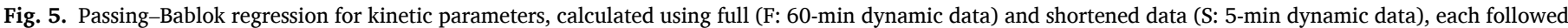

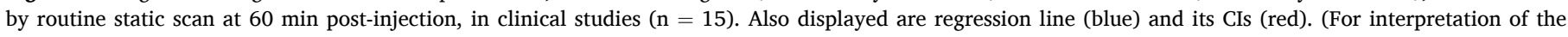
references to colour in this figure legend, the reader is referred to the web version of this article.)

duration equal or more than $5 \mathrm{~s}$ (i.e. $5,10,15,20,24$ and $30 \mathrm{~s}$ ) in the proposed method (5-min dynamic data followed by static data at 60 -min post-injection). The early frame durations of 5,24 and $30 \mathrm{~s}$ generally produced the most biased kinetic parameters. In agreement with Häggström et al. [14], $\mathrm{K}_{\mathrm{i}}$ shows the lowest change in bias and SD, and it is independent on early frame sampling for frames durations of 5-20 s. Overally, an early frame sampling of 10 -sec was found to minimize the bias and SD in kinetic parameters.

In the clinical studies, strong correlations were found between all corresponding estimated parameters as calculated using the proposed protocol and full dynamic data, respectively (Table 2). Passing-Bablok regression revealed no significant differences. However, except for $\mathrm{K}_{1}$ which had very strong fits, larger residual SD with larger 95\% confidence intervals (CIs) were observed for the other parameters.

One of the limitations of our work is the limited axial coverage, a single bed of $15 \mathrm{~cm}$ axial length, which restricts dynamic scan to a specific part of the body. Therefore our protocol can be useful for monitoring response assessment based on the approximately known location of the pathology of interest. Moreover, with the advent of long axial FOV total-body PET scanners, such a proposed protocol can provide full kinetic analysis in much less time compared with standard dynamic protocols.

\section{Conclusion}

To overcome limitations of long duration imaging in conventional dynamic FDG PET, we proposed a method consisting of single-bed-ofinterest as short as 5-min dynamic data (with an optimized sampling scheme), followed by 3-min/bed whole-body routine clinical PET imaging at 60 -min post-injection. Both simulation and clinical studies indicate that all kinetic parameters, could be reliably estimated from the proposed acquisitions with shortened dynamic PET data, enabling quantification of kinetic parameters as well as generation of SUV or SUR images.

\section{Declaration of Competing Interest}

The authors declare that they have no known competing financial interests or personal relationships that could have appeared to influence the work reported in this paper.

\section{Acknowledgments}

The authors wish to gratefully acknowledge the head of the Research Center for Nuclear Medicine at Shariati Hospital, Dr. Mohammad Eftekhari, and Center technologists especially Ms. Mersedeh Mokri.

\section{Ethical approval}

All procedures performed were in accordance with the ethical standards of the internal institution's ethical committee and with the 1964 Helsinki declaration and its later amendments or comparable ethical standards.

\section{Informed consent}

Informed consent was obtained from all individual participants for whom identifying information is included in this article according to the committee's recommendations.

\section{Appendix A. Supplementary data}

Supplementary data to this article can be found online at https://doi. org/10.1016/j.ejmp.2020.11.004. 


\section{References}

[1] Chandra R, Rahmim A. Nuclear medicine physics. 8th ed. Philadelphia: Lippincott Williams \& Wilkins; 2017.

[2] Keyes Jr JW. SUV: standard uptake or silly useless value? J Nucl Med 1995;36: 1836-9.

[3] van den Hoff J, Oehme L, Schramm G, Maus J, Lougovski A, Petr J, et al. The PETderived tumor-to-blood standard uptake ratio (SUR) is superior to tumor SUV as a surrogate parameter of the metabolic rate of FDG. EJNMMI Res 2013;3:77. https:// doi.org/10.1186/2191-219X-3-77.

[4] Kotasidis FA, Tsoumpas C, Rahmim A. Advanced kinetic modelling strategies: towards adoption in clinical PET imaging. Clin Trans Imaging 2014;2:219-37.

[5] Gupta N, Gill H, Graeber G, Bishop H, Hurst J, Stephens T. Dynamic positron emission tomography with F-18 fluorodeoxyglucose imaging in differentiation of benign from malignant lung/mediastinal lesions. CHEST J 1998;114:1105-11.

[6] Sugawara Y, Zasadny KR, Grossman HB, Francis IR, Clarke MF, Wahl RL. Germ Cell Tumor: Differentiation of Viable Tumor, Mature Teratoma, and Necrotic Tissue with FDG PET and Kinetic Modeling 1. Radiology 1999;211:249-56.

[7] Cheebsumon P, Velasquez LM, Hoekstra CJ, Hayes W, Kloet RW, Hoetjes NJ, et al Measuring response to therapy using FDG PET: semi-quantitative and full kinetic analysis. Eur J Nucl Med Mol Imaging 2011;38:832-42.

[8] Karakatsanis NA, Lodge MA, Tahari AK, Zhou Y, Wahl RL, Rahmim A. Dynamic whole body PET parametric imaging: I. Concept, acquisition protocol optimization and clinical application. Phys Med Bio 2013;58:7391-418.

[9] Torizuka T, Nobezawa S, Momiki S, Kasamatsu N, Kanno T, Yoshikawa E, et al. Short dynamic FDG-PET imaging protocol for patients with lung cancer. Eur J Nucl Med 2000;27:1538-42.

[10] Visser EP, Kienhorst LB, de Geus-Oei L-F, Oyen WJ. Shortened dynamic FDG-PET protocol to determine the glucose metabolic rate in non-small cell lung carcinoma. In: 2008 IEEE Nuclear Science Symposium Conference Record; 2008. p. 4455-8.

[11] Monden T, Kudomi N, Sasakawa Y, Yamamoto Y, Kawai N, Nishiyama Y. Shortening the duration of [18F] FDG PET brain examination for diagnosis of brain glioma. Mol Imag Biol 2011;13:754-8.

[12] Liao W-H, Lange K, Bergsneider M, Huang S-C. Optimal design in dynamic PET data acquisition: a new approach using simulated annealing and component-wise Metropolis updating. IEEE Trans Nucl Sci 2002;49:2291-6.

[13] Li X, Feng D, Wong K-P. A general algorithm for optimal sampling schedule design in nuclear medicine imaging. Comput Methods Programs Biomed 2001;65:45-59.

[14] Häggström I, Axelsson J, Schmidtlein CR, Karlsson M, Garpebring A, Johansson L, et al. A Monte Carlo study of the dependence of early frame sampling on uncertainty and bias in pharmacokinetic parameters from dynamic PET. J Nucl Med Technol 2015;43:53-60.

[15] Segars WP, Lalush DS, Tsui BM. Modeling respiratory mechanics in the MCAT and spline-based MCAT phantoms. IEEE Trans Nucl Sci 2001;48:89-97.

[16] Dimitrakopoulou-Strauss A, Georgoulias V, Eisenhut M, Herth F, Koukouraki S, Mäcke HR, et al. Quantitative assessment of SSTR2 expression in patients with nonsmall cell lung cancer using68Ga-DOTATOC PET and comparison with18F-FDG PET. Eur J Nucl Med Mol Imaging 2006;33:823-30.

[17] Jan S, Comtat C, Strul D, Santin G, Trebossen R. Monte Carlo simulation for the ECAT EXACT HR+ system using GATE. IEEE Trans Nucl Sci 2005;52:627-33.

[18] Thielemans K, Tsoumpas C, Mustafovic S, Beisel T, Aguiar P, Dikaios N, et al. STIR: software for tomographic image reconstruction release 2. Phys Med Biol 2012;57: 867.
[19] Jan S, Santin G, Strul D, Staelens S, Assie K, Autret D, et al. GATE: a simulation toolkit for PET and SPECT. Phys Med Biol 2004;49:4543.

[20] Häggström I, Schmidtlein CR, Karlsson M, Larsson A. Compartment modeling of dynamic brain PET-The impact of scatter corrections on parameter errors. Med Phys 2014;41:111907.

[21] Loening AM, Gambhir SS. AMIDE: a free software tool for multimodality medical image analysis. Mol Imaging 2003;2:131-7.

[22] Hoekstra C, Hoekstra O, Lammertsma A. On the use of image-derived input functions in oncological fluorine-18 fluorodeoxyglucose positron emission tomography studies. Eur J Nucl Med 1999;26:1489-92.

[23] Vriens D, de Geus-Oei L-F, Oyen WJ, Visser EP. A curve-fitting approach to estimate the arterial plasma input function for the assessment of glucose metabolic rate and response to treatment. J Nucl Med 2009;50:1933-9.

[24] Rowe SP, Solnes LB, Yin Y, Kitchen G, Lodge MA, Karakatsanis NA, et al. Imager4D: New Software for Viewing Dynamic PET Scans and Extracting Radiomic Parameters from PET Data. J Digit Imaging 2019;32:1071-80.

[25] Passing H, Bablok W. A new biometrical procedure for testing the equality of measurements from two different analytical methods. Application of linear regression procedures for method comparison studies in clinical chemistry, Part I. Clin Chem Lab Med 1983;21:709-20.

[26] Strauss LG, Dimitrakopoulou-Strauss A, Haberkorn U. Shortened PET data acquisition protocol for the quantification of 18F-FDG kinetics. J Nucl Med 2003; 44:1933-9.

[27] Strauss LG, Pan L, Cheng C, Haberkorn U, Dimitrakopoulou-Strauss A. Shortened acquisition protocols for the quantitative assessment of the 2-tissue-compartment model using dynamic PET/CT 18F-FDG studies. J Nucl Med 2011;52:379-85.

[28] Rahmim A, Lodge MA, Karakatsanis NA, Panin VY, Zhou Y, McMillan A, et al Dynamic whole-body PET imaging: principles, potentials and applications. Eur J Nucl Med Mol Imaging 2019;46:501-18.

[29] Zaker N, Kotasidis F, Garibotto V, Zaidi H. Assessment of Lesion Detectability in Dynamic Whole-Body PET Imaging Using Compartmental and Patlak Parametric Mapping. Clin Nucl Med 2020.

[30] Kotasidis FA, Garibotto V, Zaidi H. Hybrid whole-body dynamic TOF PET imaging for simultaneous estimation of compartmental and Patlak parametric maps from continuous bed motion data. In: 2016 IEEE Nuclear Science Symposium Medical Imaging Conference and Room-Temperature Semiconductor Detector Workshop (NSS/MIC/RTSD); 2016. p. 1-2. https://doi.org/10.1109/NSSMIC.2016.8069384.

[31] Kotasidis FA, Manari M, Garibotto V, Zaidi H. Joint optimization of kinetic modelling and CBM acquisition parameters in hybrid whole-body dynamic PET imaging. In: 2017 IEEE Nuclear Science Symposium and Medical Imaging Conference (NSS/MIC); 2017. p. 1-3. https://doi.org/10.1109/ NSSMIC.2017.8533062.

[32] Schierz J-H, Opfermann T, Steenbeck J, Lopatta E, Settmacher U, Stallmach A, et al. Early dynamic 18F-FDG PET to detect hyperperfusion in hepatocellular carcinoma liver lesions. J Nucl Med 2013;54:848-54.

[33] Nakajima R, Abe K, Kondo T, Tanabe K, Sakai S. Clinical role of early dynamic FDG-PET/CT for the evaluation of renal cell carcinoma. Eur Radiol 2016;26: $1852-62$.

[34] Mullani NA, Herbst RS, O'Neil RG, Gould KL, Barron BJ, Abbruzzese JL. Tumor blood flow measured by PET dynamic imaging of first-pass 18F-FDG uptake: a comparison with 150-labeled water-measured blood flow. J Nucl Med 2008;49: 517-23. 